\title{
Binary beryllium pnictides: ordered and disordered coloring variants of the diamond structure
}

\author{
A. Feige, M. Michak, M. Grauer, D. Günther, L. Staab, C. Benndorf, O. Oeckler \\ Leipzig University, Faculty of Chemistry and Mineralogy, Leipzig, Germany \\ oliver.oeckler@gmx.de
}

Even after decades of solid-state research, there are intriguing binary systems lacking investigation, even exclusively with main group elements. For instance, there are significantly fewer investigations on beryllium compounds than on any other class of light-element materials, even though beryllium-containing phases feature interesting properties for basic and applied research.[1] Owing to its toxicity, efforts to understand the chemistry of Be are rather rare. However, the limited knowledge present promises a rich and unusual structural chemistry. The few results concerning Be compounds with group 15 elements include the disordered diamond-like structure of $\mathrm{BeP}_{2}$.[2] Yet, the true building blocks, i.e. the arrangement of polyphosphide anions, remained elusive with respect to the description of the average structure. Preliminary work on $\mathrm{BeAs}_{2}$ and $\mathrm{BeSb}_{2}$ indicates related structures for both compounds;[3] however, this information is only based on qualitative evaluation of powder X-ray diffraction data. Precise structural data require very accurate diffraction data due to the large difference in scattering factors. Despite the simple stoichiometry, a complete structural analysis proved difficult as the crystals obtained are by far too small for data collection using laboratory diffractometers. We now employed a combined approach using microfocused synchrotron radiation, electron diffraction and HRTEM.

Synchrotron data of a microcrystal of $\mathrm{BeSb}_{2}$ reveal a coloring variant of the cubic diamond structure (Fig. 1). The corresponding tetragonal superstructure contains twisted chains of $\mathrm{Sb}$ atoms interconnected by Be atoms with all atoms showing a distorted tetrahedral coordination. The conformation of the polyanion corresponds to the Ge substructure in $\mathrm{Li}_{{ }_{3}} \mathrm{AgGe}_{2}$. [4] This indicates chemical bonding according to a Zintl phase with a "sulfur-like" Sb- polyanion (comparable to $\mathrm{Ge}^{2-}$ ). Yet, $\mathrm{BeSb}_{2}$ can also be viewed as a Grimm-Sommerfeld semiconductor with an average valence electron concentration of 4. Compared to $\mathrm{Be}_{13} \mathrm{Sb}$, which features $\mathrm{Be}_{12}$ icosahedrons in analogy to the $\mathrm{NaZn}_{13}$ type, the bonding situation changes from quasi-molecular entities to typical semiconductors upon varying the relative Be content. Hypothetical intermediate structures may exhibit rather unusual chemical bonding.

For $\mathrm{BeP}_{2}$ and $\mathrm{BeAs}_{2}$, our investigations have confirmed the disordered diamond-like / sphalerite-like structures according to the average structures in literature, which can be refined in space group I4 1 /amd.[2,3] Diffraction patterns (both with X-rays and electrons, Fig. 2) exhibit pronounced diffuse streaks that indicate stacking disorder. Synchrotron data were collected from microcrystallites on TEM grids that were pre-characterized by electron microscopy. Both the evaluation of synchrotron diffraction data and HRTEM imaging reveal the nature of the disorder and the local structure of the polyanions. Stacking probabilities were derived by simulation diffraction patterns. The degree of ordering varies: diffuse streaks can be almost uniform but, especially in the case of BeAs2, they may also approach a superstructure.

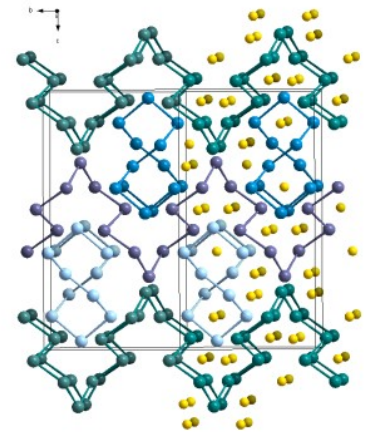

Fig. 1: Crystal structure of $\mathrm{BeSb}_{2}$

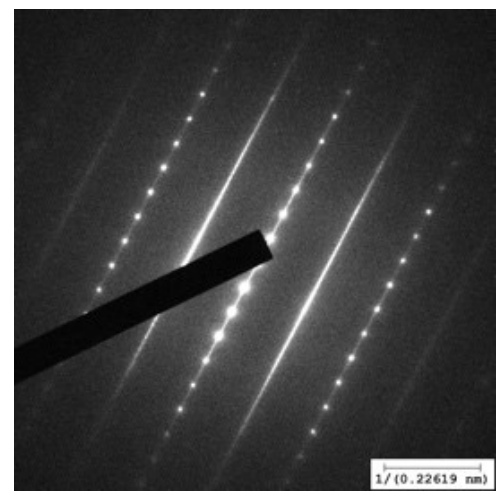

Fig. 2: SAED pattern of BeAs2

[1] M. R. Buchner, R. Pöttgen, H. Schmidbaur (2020). Z. Naturforsch. 75b, 403.

[2] P. L'Haridon, J. David, J. Lang, E. Parthé (1976). J. Solid State Chem. 19, 287.

[3] R. Gerardin, J. Aubry (1976). J. Solid State Chem. 17, 239.

[4] A. Henze, V. Hlukhyy, T. F. Fässler (2015). Inorg. Chem. 54, 1152.

Keywords: beryllium pnictides; disordered structures; synchrotron radiation Acta Cryst. (2021), A77, C550 